Ann. Sci. forest., I975, 32 (2), I3I-I34.

NOTE TECHNIQUE

\title{
PRÉCISIONS DES MESURES DE SUPERFICIES PAR COMPTAGE DE POINTS
}

\author{
J. BOUCHON \\ Station de Sylviculture et Production, \\ Centre national de Recherches forestières, I. N. R. A., \\ Champenoux, 54280 Seichamps
}

\begin{abstract}
RÉSUMÉ
L'auteur présente une méthode de calcul de l'erreur dans les estimations de superficie par comptage de points.

La mesure de superficies par comptage de points est un problème théoriquement compliqué et pratiquement assez simple. Les applications forestières peuvent être nombreuses, par exemple : mesure de surfaces de parcelles forestières, de projection au sol de houppiers, de sections de fût d'arbres, etc. Cet article se limitera à l'aspect pratique ; pour la théorie le lecteur peut se référer à G. Matheron (1970) : La théorie des variables régionalisées et ses applications ( ${ }^{1}$ ).
\end{abstract}

\section{I. - MESURE}

Pour obtenir une estimation non biaisée d'une surface, il suffit d'utiliser une grille régulière et de compter le nombre de points se trouvant à l'intérieur de la surface considérée. Le maillage de la grille peut être rectangulaire ou triangulaire. La figure $\mathrm{I}$ montre une surface comprise entre deux cercles ayant respectivement comme rayons $5,64 \mathrm{~cm}$ et $2,82 \mathrm{~cm}$ : la superficie exacte est donc de $75 \mathrm{~cm}^{2}$.

(1) Les cahiers du Centre de morphologie mathématique, Fascicule 5. École Nationale Supérieure des Mines de Paris. 35, Rue Saint-Honoré, 77305 Fontainebleall. 
En appliquant une grille carrée de $\mathrm{I}$ cm de côté, on compte $\mathrm{N}=77$ points, ce qui donne une estimation de la superficie

$$
\mathrm{S}=77 \mathrm{~cm}^{2}
$$

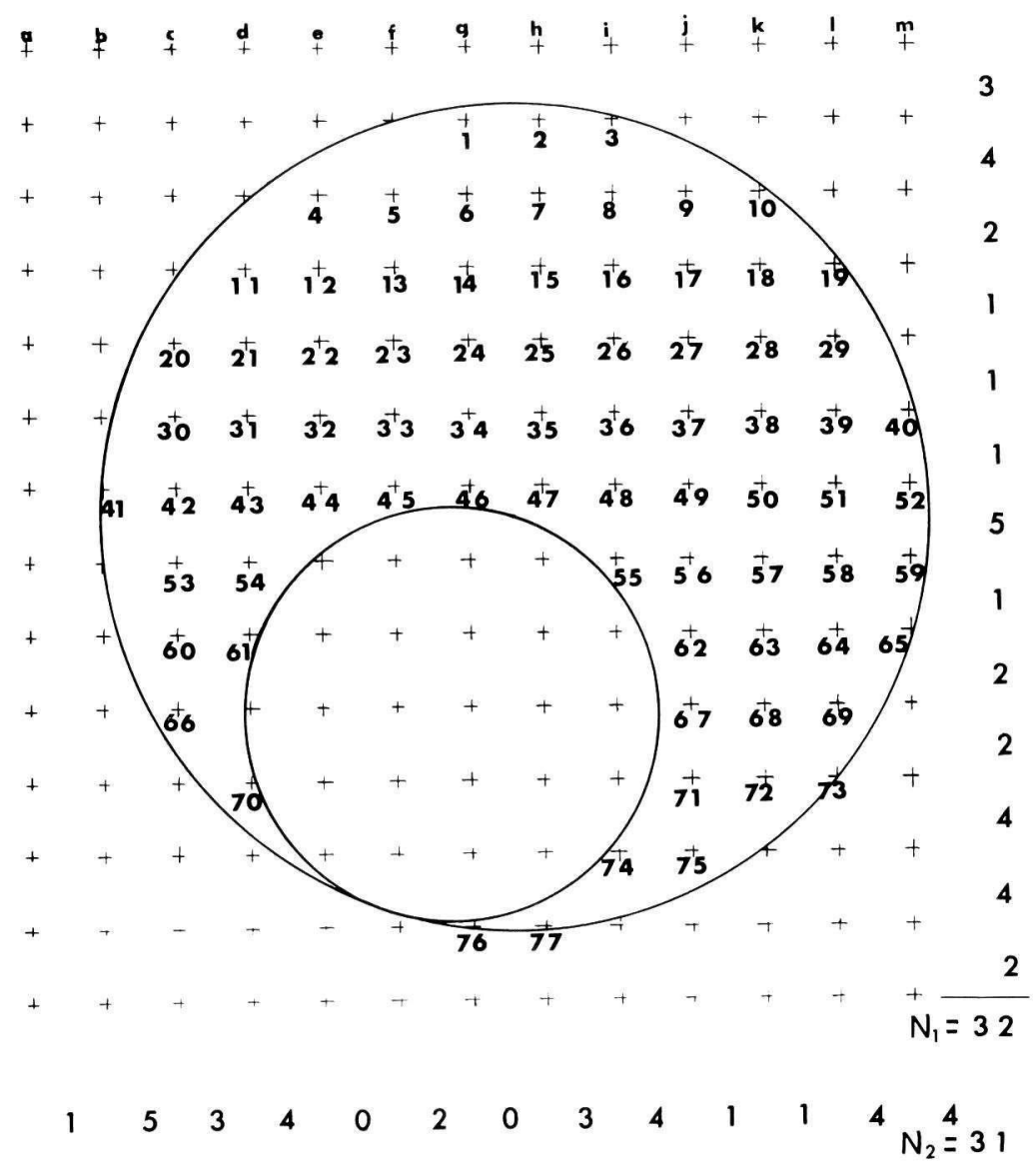

FiG. I

(Figure réduite à l'échelle 3/4)

\section{2. - PRÉCISION}

Pour déterminer la précision, un maillage carré, ou rectangulaire, est nécessaire. Deux nombres sont à calculer :

$\mathrm{N}_{l}$ pour les lignes et $\mathrm{N}_{c}$ pour les colonnes.

Règle pour le calcul de $N_{\mathrm{c}}$ : dans chaque colonne un certain nombre de points appartiennent à la surface ; pour la figure I si l'on va de la gauche vers la droite, les nombres de points successivement rencontrés par colonne sont: o, I, 6, 7, 5, 5, 7, 7, 8, I0, 9, 8, 4, o.

Pour chacun des couples constitués de 2 colonnes voisines, on compte le nombre de points apparus ou disparus. 
Exemples:

- Colonnes $a / b$ : le point ${ }_{4} \mathrm{I}$ est apparu : la contribution de ces 2 colonnes au calcul de $\mathrm{N}_{c}$ est donc $\mathbf{I}$.

- Colonnes $b / c$ : les points 20, 30, 53, 60, 66 n'ont pas d'homologues dans la colonne $b$; la contribution de ces 2 colonnes à $\mathrm{N}_{c}$ est donc 5 ,

- colonnes $c / d$ : les points i i et 70 de la colonne $d$ sont apparus ; le point 66 a disparu. La contribution au calcul de $\mathrm{N}_{c}$ est donc $3 \ldots$ etc.,

- colonnes $h / i$ : les points 77 de la colonne $h$ et 55,74 de la colonne $i$ n'ont pas d'homologues dans l'autre colonne : la contribution au calcul de $\mathrm{N}_{c}$ est donc $3 \ldots$ etc.

La valeur de $\mathrm{N}_{\boldsymbol{c}}$ est la somme des nombres ainsi obtenus; ici $\mathrm{N}_{c}=3 \mathbf{I}$.

En opérant de même pour les lignes on trouve $\mathrm{N}_{l}=32$.

On appelle $N_{1}$ la plus grande de ces deux valeurs et $N_{2}$ la plus petite.

$$
\text { ici : } \begin{aligned}
\mathrm{N}_{1} & =\mathrm{N}_{l}=32 \\
\mathrm{~N}_{2} & =\mathrm{N}_{c}=3 \mathrm{I}
\end{aligned}
$$

La formule suivante permet de calculer la valeur de la variance $\sigma_{s}^{2}$ de l'estimation $\mathrm{S}$ :

$$
\frac{\sigma_{s}^{2}}{\mathrm{~S}^{2}}=\frac{\mathrm{I}}{\mathrm{N}^{2}}\left(\frac{\mathrm{N}_{2}}{\mathrm{I} 2}+0,0305 \frac{\mathrm{N}_{1}^{2}}{\mathrm{~N}_{2}}\right)
$$

avec $\mathrm{N}_{2} \leqslant \mathrm{~N}_{1}$.

Cette formule correspond en réalité aux deux premiers termes de la formule donnée par la théorie (G. Matheron, I970).

Le calcul donne $\sigma_{s}^{2}=3,59$

Soit un écart-type de $\mathrm{I}, 894$

L'estimation de la surface au seuil de o,05 est donc

$$
\mathrm{S}=77 \pm 3,7 \mathrm{~cm}^{2}
$$

\section{3. - CALCUL DIRECT ÉT VÉRIFICATION}

- On a lancé 6 fois la figure sur un papier millimétré pour avoir une idée de la variabilité de l'estimation de $\sigma_{s}^{2}:$ les 6 valeurs trouvées sont :

$$
3.59-3,37-3,22-3,44-3,22-3,50
$$

- On a lancé 30 fois la figure pour avoir une mesuve directe de $\sigma_{s}^{2}$ : les 30 valeurs de $\mathrm{N}$ ainsi trouvées avaient comme moyenne 74,5 et comme variance 3,52 . Cette mesure concorde parfaitement avec les 6 valeurs fournies par la théorie.

\section{CONCLUSION}

Une méthode de calcul de l'erreur dans les estimations de superficies par comptage de points est présentée avec la formule donnant $\sigma_{s}^{2} / \mathrm{S}^{2}$.

Les calculs faits avec cette méthode sont confrontés à ceux que donne une simulation directe : les résultats sont concordants. 


\section{SUMMARY}

PRECISION OF AERA ESTIMATIONS CALCULATED BY A SYSTEMATIC POINT GRID

This note gives a method to calculate the precision of aera estimations calculated by a systematic point grid. 